\title{
Low-dimensional long-range topological structure in the QCD vacuum *
}

\author{
I. Horváth ${ }^{\mathrm{a}}$, S.J. Dong ${ }^{\mathrm{a}}$, T. Draper ${ }^{\mathrm{a}}$, F.X. Lee ${ }^{\mathrm{bc}}$, K.F. Liu ${ }^{\mathrm{a}}$, N. Mathur ${ }^{\mathrm{a}}$, J.B. Zhang ${ }^{\mathrm{d}}$, H.B. Thacker \\ ${ }^{a}$ Department of Physics \& Astronomy, University of Kentucky, Lexington, KY 40506, USA \\ ${ }^{\mathrm{b}}$ Center for Nuclear Studies, George Washington University, Washington, DC 20052, USA \\ ' Jefferson Lab, 12000 Jefferson Avenue, Newport News, VA 23606, USA \\ ${ }^{\mathrm{d}}$ CSSM and Dept. of Physics and Math. Physics, University of Adelaide, Adelaide, SA 5005, Australia \\ ${ }^{\text {e}}$ Department of Physics, University of Virginia, Charlottesville, VA 22901, USA
}

Lattice topological charge associated with Ginsparg-Wilson fermions exhibits generic topological stability over quantum ensemble of configurations contributing to the QCD path integral. Moreover, the underlying chiral symmetry leads to the suppression of ultraviolet noise in the associated topological charge densities ("chiral smoothing"). This provides a solid foundation for the direct study of the role of topological charge fluctuations in the physics of QCD vacuum. Using these tools it was recently demonstrated that: (a) there is a well-defined spacetime structure (order) in topological charge density (defined through overlap fermions) for typical configurations contributing to QCD path integral; (b) this fundamental structure is low-dimensional, exhibiting sign-coherent behavior on subsets of dimension less than four and not less than one; (c) the structure has a long-range global character (spreading over maximal space-time distances) and is built around the locally one-dimensional network of strong fields (skeleton). In this talk we elaborate on certain aspects and implications of these results.

The aim of this talk is to emphasize three messages (given in the abstract) from Ref. [1]. To appreciate the relevance of these messages, it is useful to recall that while the topology of gauge fields is presumed to be associated with interesting non-perturbative physics in QCD, the progress in making a detailed connection has been quite slow. One possible path toward the breakthrough uses the lattice, where one has direct access to typical configurations contributing to the regularized QCD path integral. In principle then, one could study the space-time structure of topological charge density for typical configurations, and identify the characteristics of this structure that have direct bearing on the physical phenomena in question. However, there used to be several difficult obstacles as well as questions of principle preventing the realization of such a direct program.

(i) Thinking in terms of topology implies the availability of quantities that are stable under

\footnotetext{
${ }^{*}$ Talk presented by I. Horváth, at Lattice 2003 Symposium, Tsukuba, Japan.
}

continuous deformations. The simplest topological field in the continuum is the topological charge density (TChD), whose associated global charge is stable under the local changes of the gauge field $\left(\delta Q / \delta A_{\mu}(x)=0\right)$ for sufficiently smooth fields. The basic problem on the lattice is that it is nontrivial to define a local topological field. In particular, naive discretizations of $F \tilde{F}(x)$ are not stable at all (see however Ref. [2]).

(ii) Even if a lattice topological field, stable over some class of lattice gauge fields is available, there still might be a deep problem. The point is that we are interested in the quantum theory defined by the path integral. To study the relevance topology in this setting, one should thus ensure that topological stability is guaranteed for typical configurations (not necessarily smooth) from the quantum QCD ensemble.

(iii) Even with points (i) and (ii) fulfilled, we might not gain much since it is possible that there is no well-defined space-time structure in TChD. Indeed, the mere existence of the structure at the configuration level is a subject of debate. While 
the action favours infrared fluctuations, there are many more possibilities for ultraviolet ones. In fact, for the commonly used lattice gauge actions there is an extensive experience indicating that when naive $\mathrm{TChD}$ operators are used, the ultraviolet noise dominates, and there is no detectable space-time order at all. However, without the order, we can not learn anything about the vacuum, and the approach outlined above would fail. This is the problem of ultraviolet dominance or the "entropy" problem.

The point (i) is elegantly resolved by using the TChD associated with GW fermions (see also Ref. [2]). Indeed, for lattice Dirac operator satisfying $\gamma_{5} D \gamma_{5}=D^{\dagger}$ and $\left\{D, \gamma_{5}\right\}=D \gamma_{5} D$, the pseudoscalar operator $q_{x}=-\operatorname{tr} \gamma_{5}(1-$ $\left.\frac{1}{2} D_{x, x}\right)$ represents a well-defined local topological charge density [34], whose integral is stable under generic local changes of the gauge field [5]. Morover, this $q_{x}$ is generically stable for all backgrounds for which $D$ itself is well-defined! The accumulated experience with the overlap Dirac operator 6] (used here) indicates that this includes typical configurations from currently used QCD ensembles, thus fulfilling (ii).

Conceptually the most important result of Ref. [1 is that the point (iii) is also fulfilled if $q_{x}$ associated with overlap fermions is used, i.e. the conclusion (a) of the abstract holds and the problem of ultraviolet dominance is solved. We emphasize that this is a qualitatively new result since, contrary to previous studies of structure which always involved some particular processing of gauge configurations, our approach is completely unbiased and gauge invariant: we just use the appropriate local operator to measure $\mathrm{TChD}$. Also note that the structure uncovered this way is the fundamental structure in the sense that (while suppressing ultraviolet noise) it includes the fluctuations at all scales up to the lattice cutoff. It can thus have manifestations reflecting both short and long-distance physics.

An important clue in finding this structure is the old observation [7, that in the continuum $\langle q(x) q(0)\rangle \leq 0,|x|>0$. As pointed out in Ref. [8], this suggests that the fundamental structure cannot involve topological charge concentrated mainly in sign-coherent 4 -d lumps. (The

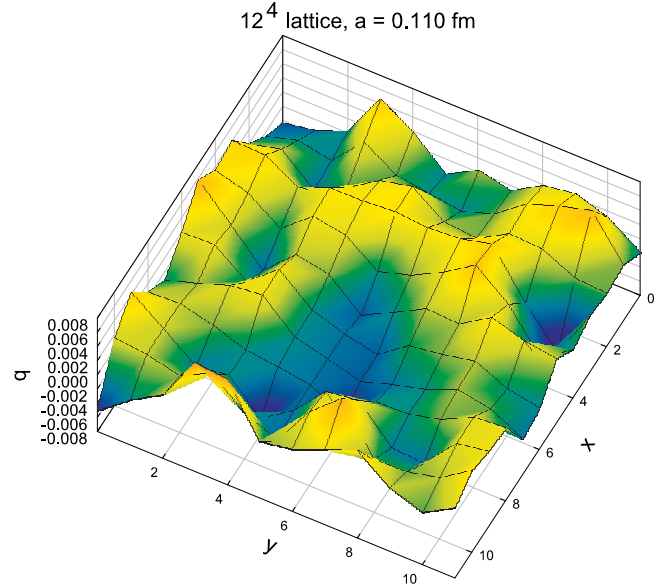

results of [1] not only demonstrate this but also suggest that in QCD the 4-d sign-coherent structure does not occur at all.) Given that, the starting idea of Ref. [1] is that the ordered structure could manifest itself by the enhanced signcoherence present on low-dimensional subsets of 4-d Euclidean space. Indeed, the negativity of the correlator could then be satisfied in an ordered manner by embedding structures with alternating sign in 4-d space. The existence of such order has in turn been demonstrated 1 .

Using sign-coherence as a basic guide, it was found that about $80 \%$ of space-time is covered by two connected folded "sheets" (of opposite sign) built from 3-d elementary coherent cubes. The sheets thus essentially fill the space-time. To get the sense of how this looks, we plot above $q_{x}$ on a (generic) 2 -d plane of a $12^{4}$ configuration with Wilson gauge action $(\beta=5.91)$. The enhancement of the structure is obvious with linear "ridges" running across the whole system. Also, the low-dimensionality is nicely reflected in this generic picture. Indeed, if $\Omega$ is the base manifold and $\Omega_{1}, \Omega_{2} \subset \Omega$, then $\operatorname{dim}\left(\Omega_{1} \cap \Omega_{2}\right)=$ $\operatorname{dim}\left(\Omega_{1}\right)+\operatorname{dim}\left(\Omega_{2}\right)-\operatorname{dim}(\Omega)$ generically. Identifying $\Omega$ with the 4 -d space-time torus, $\Omega_{1}$ with the 2-d torus of the section plane, and $\Omega_{2}$ with the manifold of the structure, we get

$\operatorname{dim}\left(\Omega_{1} \cap \Omega_{2}\right)=\operatorname{dim}\left(\Omega_{2}\right)-2$

Consequently, if $\Omega_{2}$ is a 3 -d hypersurface, we should generically see extended 1-d regions of 
sign-coherence. This is indeed what is observed. Thus, at the lattice cutoff of about $2 \mathrm{GeV}$ the sheets behave as 3-d hypersurfaces. While the precise local dimension of the maximal signcoherent regions in the continuum limit remains unknown, the available data allow us to conclude part (b) of the abstract [1].

Apart from low-dimensional nature, the striking property of the TChD structure is its longrange and global character. Indeed, the sheet appears to behave as one whole and due to connectedness and the space-filling feature, one can reach maximal possible distances on the paths within the same sheet. Moreover, this remains true even if one considers only regions occupied by strong fields. In particular, there is a well-defined minimal fraction of space-time $(\approx 18 \%)$ containing the most intense TChD while still exhibiting this super-long-distance (SLD) property [1]. We refer to the substructure defined this way as a skeleton since the rest of the sign-coherent sheet is built around it. An intriguing property of the skeleton is that it is locally 1-dimensional [1, and thus can be viewed as a network of world-lines for pointlike objects.

The above properties of the fundamental structure suggest the following two propositions deserving further examination: (A) The propagation of light quarks and pions is facilitated by the long-range (SLD) skeleton. One should thus try to relate the microscopic understanding of spontaneous chiral symmetry breaking ( $\mathrm{SChSB})$ to the properties of this locally 1-dimensional structure. An interesting clue in making this connection is the fact that the motion of quarks restricted to 1-dimensional manifold naturally leads to nonzero density of eigenmodes near zero, and hence SChSB (see e.g. some related discussion 10]). (B) Global topological stability in quantum QCD configurations cannot be understood in terms of stability of its subsets, i.e. the global charge will not result as a sum of contributions from welldefined separate pieces. The global stability can only be understood if one considers the structure (or its SLD subset such as skeleton) as a whole. This can be considered a generalization of the result demonstrated in Refs. 89, i.e. that topological charge does not appear in quantized lumps of unit topological charge (even at low energy).

Finally, we comment on the mechanism leading to the resolution of the entropy problem (iii). The new element brought in by the imposition of chiral symmetry is that the GW operators are nonultralocal, i.e. there is nonzero coupling among variables at arbitrarily large space-time distances. This has been proved for fermionic degrees of freedom 11, but it is expected to hold also in terms of gauge variables. The implied presence of arbitrarily extended loops (albeit with small coupling) in the definition of $q_{x}$ leads to taming of ultraviolet noise at the scale of the cutoff to a certain degree ("chiral smoothing" 9]). Moreover, the availability of these loops can help to uncover the long-range coherence (masked by ultraviolet noise) on extended low-dimensional manifolds since the loops contained inside these manifolds will contribute coherently. The results of [1] imply that such coherence exists, and is indeed exposed by chiral smoothing.

\section{REFERENCES}

1. I. Horváth et al., hep-lat/0302009 hep-lat/0212013.

2. M. Lüscher, Commun. Math. Phys. 85 (1982) 39.

3. P. Hasenfratz, V. Laliena, F. Niedermayer, Phys. Lett. B427, 125 (1998).

4. M. Lüscher, Nucl. Phys. B (Proc. Suppl.) 83, (2000) 34.

5. R. Narayanan, H. Neuberger, Nucl.Phys. B443, (1995) 305.

6. H. Neuberger, Phys. Lett B417 (1998) 141.

7. E. Seiler, I.O. Stamatescu, MPI-PAE/Pth 10/87.

8. I. Horváth et al., Phys. Rev. D67, 011501(R) (2003).

9. I. Horváth et al., Phys. Rev. D66, 034501 (2002).

10. G. Tiktopoulos, Phys. Rev. D35, 732 (1987); J.M. Cornwall, G. Tiktopoulos, Phys. Lett. B181, 353 (1986); H. Reinhardt, private communication.

11. I. Horváth, Phys. Rev. Lett. 81 (1998) 4063; I. Horváth, C.T. Balwe, R. Mendris, Nucl. Phys. B599, 283 (2001). 